\title{
Helically twisted long-period fiber gratings of YOFC single-mode fiber with optical fiber welding machine
}

\author{
Yunfeng Bai, Zelong He, Jiyuan Bai, Suihu Dang ${ }^{*}$
}

Key Laboratory of Micro Nano Optoelectronic Devices and Intelligent Perception Systems, School of Electronic Information and Engineering, Yangtze Normal University, Chongqing, 408100, China

*Corresponding author: dangsuihu@qq.com

\begin{abstract}
The single-mode fiber provided by YOFC inc is employed for spiral processing by commercial welding machine. It can clearly see the periods structure under the light, but there is no obvious deformation of the fiber core, cladding and surface morphology under a microscope. There is an obvious resonant peak near $1560 \mathrm{~nm}$, half peak width is about $25 \mathrm{~nm}$, the depth of the resonant peak closed to $-26 \mathrm{~dB}$, when the period is about $411 \mu \mathrm{m}$. It agrees with theoretical calculation results according to the long-period fiber grating coupled-mode theory. The resonance wavelength is caused by coupling between the fundamental mode and the $\mathrm{LP}_{14}$ mode. The responsivities of the helically twisted long-period fiber gratings (H-LPFG) for the temperature is measured, the resonance wavelength is linear with temperature, the slope is $86 \mathrm{pm} /{ }^{\circ} \mathrm{C}$. Because it is easy to inscribe by commercial welding machine, and has a strong resonance peak, it has potential applications as the temperature sensor.
\end{abstract}

Keywords: YOFC single-mode fiber, helically twisted long-period fiber gratings, fundamental mode, cladding mode, coupled-mode theory.

\section{Introduction}

Chiral fiber grating (CFG) is a kind of fiber having periodic helical refractive index modulation with the axial and angle. In 2004 [1] the CFG was reported, and since then, it has been widely studied. It has the circular polarization selection, wavelength selection, orbital angular momentum and sensing applications, which attract wide attention [ $\underline{2}-\underline{4}]$. Helically twisted long-period fiber grating (H-LPFG) is a kind of a long-period CFG. It has advantages of both long-period fiber grating and CFG at the same time.

The H-LPFGs, due to their advantages in the helical structure and long-period fiber grating, have been mainly applied to sensing the twisting rate [ $\left.\underline{5}_{-}^{-}\right]$], the refractive in$\operatorname{dex}[\underline{8}]$, the applied stress [9] , temperature $[\underline{9}, \underline{10}]$, and to generate orbital angular momentum [11-13]. In 2014, GAO et al. processed CFG of a multipoint phase shift with 
$\mathrm{CO}_{2}$ laser. The twist sensitivity of CFG is $1.959 \mathrm{~nm} /(\mathrm{rad} / \mathrm{m})$, and it has lower temperature cross sensitivity []. In 2016, WANG and Li proposed a new method enabling fabrication of H-LPFGs in a thinned fiber with a diameter smaller than several tens of micrometers. This method has been applied to the refractive index measurement [ $[$ ] In 2017, BING et al. produced H-LPFGs by the optical fiber welding machine with programming, in which the strain sensitivity is $1.88 \mathrm{pm} / \mu \varepsilon$ and the temperature sensitivity is $69.9 \mathrm{pm} /{ }^{\circ} \mathrm{C}$ [9]. In 2018, Fu et al. used single-mode H-LPFGs to generate 1 order vortex light [11]. In 2019, ZHAO et al. used four mode H-LPFGs to generate 2 order vortex [12].

Until now, many kinds of fibers have been spirally processed, for example, corning SMF-28 optical fiber $[\underline{13}, \underline{14}]$, less mode optical fiber $[\underline{12}, 15]$, photonic crystal fiber $[\underline{4}, \underline{16}, \underline{17}]$, partial core optical fiber [18], etc., but there are few studies about YOFC fiber single-mode. In this paper, YOFC single-mode fiber is spirally processed by a commercial welding machine, and the relationship between the period and the resonant peak is different from corning SMF-28 optical fiber presented in former reports [11]. They also studied the transmission spectra variety with temperature. The sensitivity and linearity of the resonant peak with temperature have an application potential.

\section{Experiment}

The YOFC single-mode fiber is spirally processed by a welding machine (Fujikura FSM-100p+). Firstly, the electrode discharge calibration and motor calibration are carried out on the commercial fusion splicer, when making electrode discharge calibration, $Z$ axial motor is also calibrated. The distance between electrodes is $2.5 \mathrm{~mm}$. As shown in Fig. 1, the peeled off part of the coating layer of YOFC single-mode fiber and the fiber are clamped by two optical fiber fixtures with slight tension. A special welding mode is selected. The stepping motor selects a sweep model, which means that the optical fiber fixture and the fiber between them are synchronous, moving with a constant speed, and the stepping speed is set to $0.056 \mu \mathrm{m} / \mathrm{ms}$. ZR motor is set to rotate clockwise, and the rotation speed is $0.049^{\circ} / \mathrm{ms}$. Electrode discharge power is set to $-80 \mathrm{bit}$, running time is set to $300000 \mathrm{~ms}$.

Figure 2a shows the processed H-LPFGs. The periods structure can clearly be seen under light. The period is about $411 \mu \mathrm{m}$. But as shown in Fig. $2 \mathbf{b}$, the fiber core, clad-

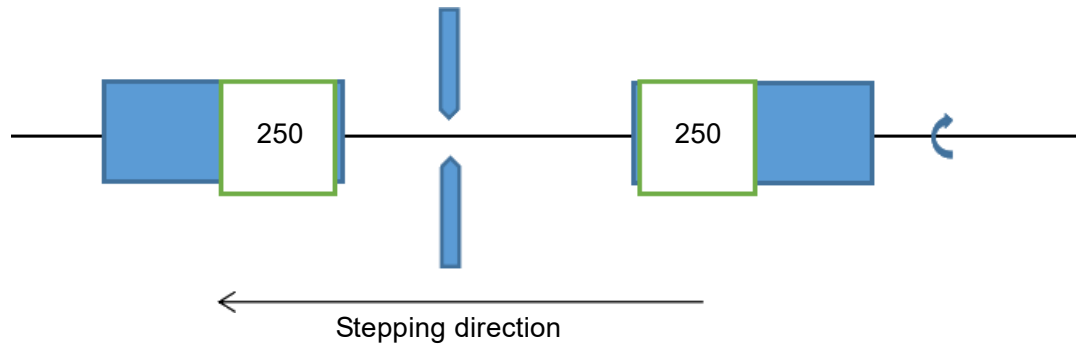

Fig. 1. Schematic of the spiral processing employing a commercial fusion splicer. 

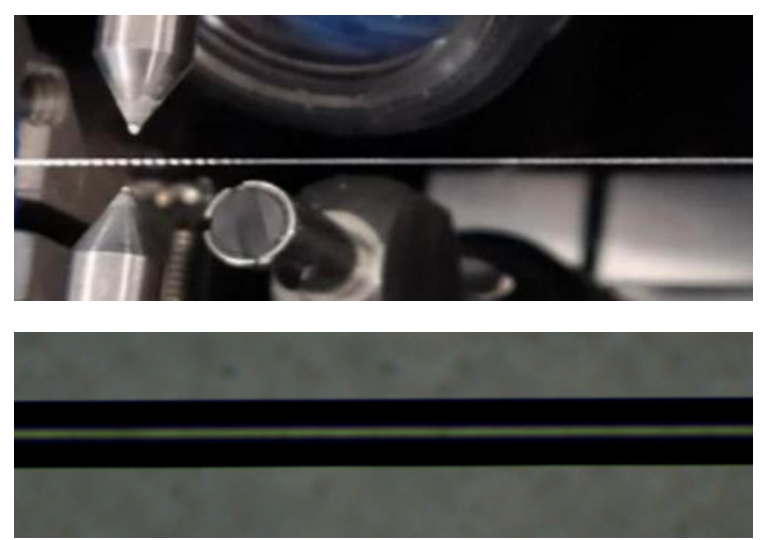

b

Fig. 2. Photograph of the spiral processed fiber (a), and microscope photograph of the spiral processed fiber (b).

ding and surface morphology of the processed H-LPFGs have no obvious changes under the microscope (Olympus CX23). It suggests that the periods structure shown in Fig. $1 \mathbf{b}$ is caused by an uneven refractive index in spiral processing. Especially, the cladding refractive index is modulated significantly, but the optical fiber has no obvious deformation. Of course, there would be some very tiny optical fiber deformations, which have little effect on transmission spectra.

The period of the H-LPFGs is equal to the pitch of helix, which can be calculated by

$$
\Lambda=360 \frac{V}{\theta}
$$

where $V$ is stepping speed of sweep mode, $\theta$ is rotation speed of ZR motor. The grating pitch of H-LPFGs is calculated by Eq. (1), which is $411.4 \mu \mathrm{m}$, and the total length has about 40 periods.

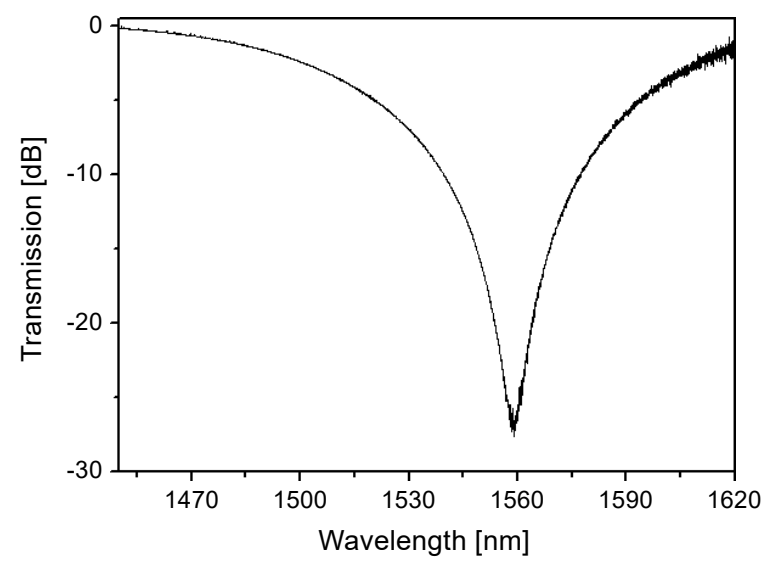

Fig. 3. The transmission spectrum of the processed H-LPFG. 
The transmission spectrum is measured. The spectrometer is Yokogawa-AQ6370D. The spectral range of a broadband light source is from 1400 to $1620 \mathrm{~nm}$. The measured transmission spectrum at room temperature is shown in Fig. 3. There is an obvious resonant peak near $1560 \mathrm{~nm}$, and half peak width is about $25 \mathrm{~nm}$. The depth of the resonant peaks is closed to $-26 \mathrm{~dB}$, and the insert losses are very small.

\section{Discussion}

According to the long-period fiber grating coupled-mode theory [19-21], the guided fundamental mode is coupled to the forward-propagating cladding modes, and leads to the resonant peak. For the single-helix H-LPFG in a conventional SMF, the phase -matching condition can be described as

$$
\begin{aligned}
& n_{\mathrm{F}}-n_{\mathrm{N}}=\frac{\lambda_{\mathrm{D}}}{\Lambda} \\
& \lambda_{\text {res }}=\frac{1}{1-\left(\sigma_{11}-\sigma_{22}\right) \frac{\Lambda}{2 \pi}} \lambda_{\mathrm{D}}
\end{aligned}
$$

where $n_{\mathrm{F}}$ and $n_{\mathrm{N}}$ are the effective refractive index of the fundamental mode and the coupled $m$-th cladding modes, respectively. $\Lambda$ is the period of the H-LPFGs. $\sigma_{11}$ and $\sigma_{22}$ are dc coupling coefficients of the fundamental mode and the coupled $m$-th cladding mode, respectively. $\lambda_{\mathrm{D}}$ is the design wavelength and $\lambda_{\text {res }}$ is the resonant wavelength. The diameter of the core, cladding, the refractive indexes of the core $n_{1}$ and cladding $n_{2}$, are $9.3 \mu \mathrm{m}, 125 \mu \mathrm{m}, 1.4628$, and 1.4573 , respectively.

As shown in Fig. 4, the effective refractive index of the fundamental mode and the cladding modes are calculated. Both the effective refractive index of the fundamental mode and the cladding modes are monotonic decreasing with the increasing of wave-

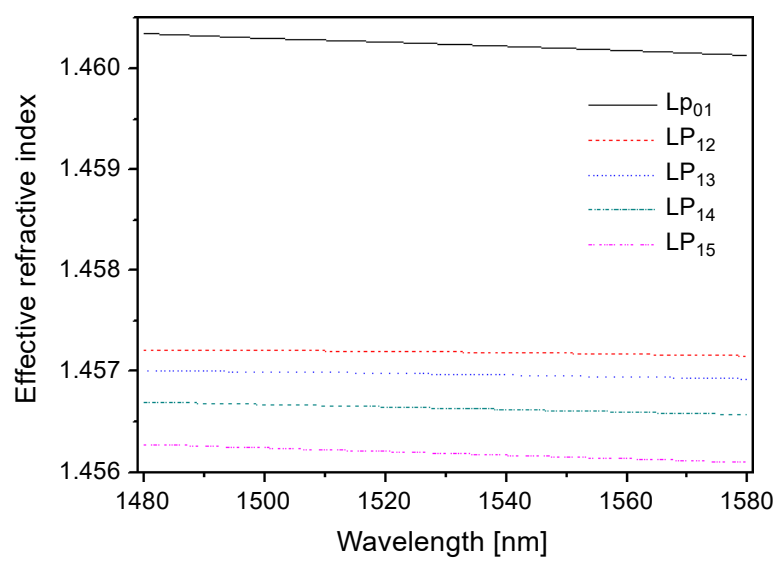

Fig. 4. The relationship between the effective refractive index and the wavelength in different modes. 


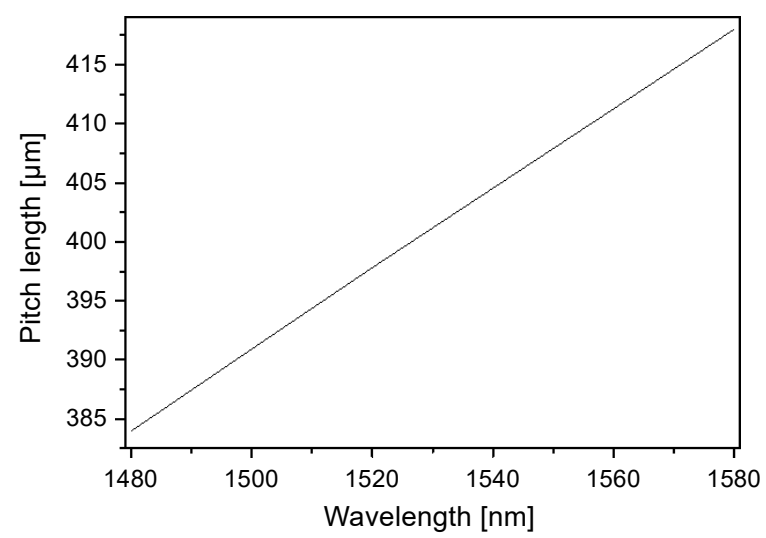

Fig. 5. Pitch length $v s$. wavelength of coupling between fundamental mode $\mathrm{Lp}_{01}$ and cladding mode $\mathrm{LP}_{14}$.

length. According to Eqs. (2) and (3), the couple between the fundamental mode and the $\mathrm{LP}_{14}$ mode can be calculated. The relation between the pitch length and the resonant wavelength is shown in Fig. 5. The difference of dc coupling coefficient between the fiber core and the cladding is 0.0008632 . The pitch length increases with the increasing of resonant wavelength. When the pitch length is $411 \mu \mathrm{m}$, the wavelength is around $1560 \mathrm{~nm}$. This experimental result coincides with Fig. 5, which means that the resonant wavelength in Fig. 3 is coupled by the fundamental mode and the 4th cladding mode. The pitch lengths of 390 and $400 \mu \mathrm{m}$ are also processed; the resonant wavelengths are around 1500 and $1530 \mathrm{~nm}$.

The responsivities of the H-LPFG for the temperature with a broadband light source and optical spectrum analyzer are measured. The temperature change from 30 to $150^{\circ} \mathrm{C}$ and the transmission spectrum are measured at every $10^{\circ} \mathrm{C}$. The results are shown in Fig. 6; the resonant wavelength increased with the increase in temperature. The fitting result is linear, the slope is $86 \mathrm{pm} /{ }^{\circ} \mathrm{C}$. Compared with Ref. [9], the single-mode fiber

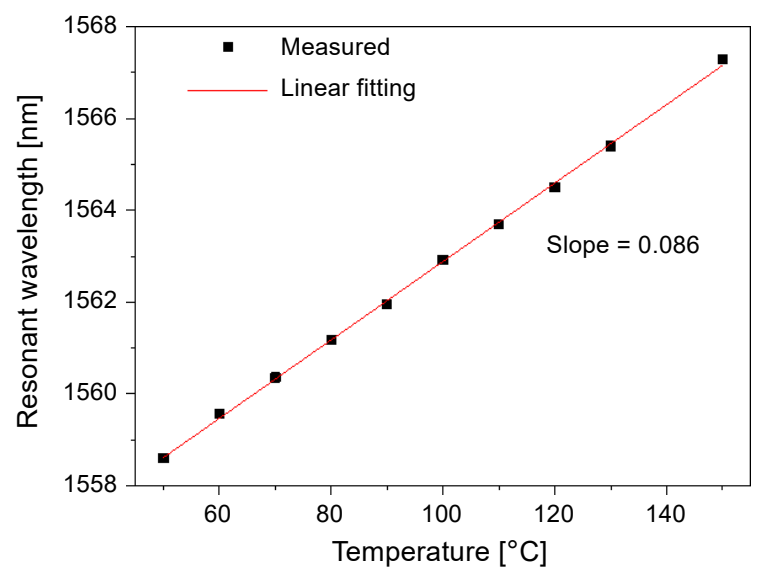

Fig. 6. Resonance wavelength $v s$. temperature. 
is different, and the coupling between the fundamental mode and the cladding mode is also different. By the use of YOFC's single-mode fiber, the slope is slightly larger than Ref. [9], and the linearity is better than Ref. [9]. Because of easy inscription process, it has potential applications in as a temperature sensor.

\section{Conclusion}

In conclusion, H-LPFGs of YOFC single-mode fiber is processed by a commercial welding machine. The periods' structure can be easily observed with a naked eye, and no significant change is seen under a microscope. The period of processed H-LPFGs is about $411 \mu \mathrm{m}$; the resonant wavelength is near $1560 \mathrm{~nm}$. The resonance wavelength is caused by coupling between the fundamental mode and the $\mathrm{LP}_{14}$ mode. The resonance wavelength $v s$. temperature is studied The resonant wavelength increased linearly with increasing temperature; the slope is $86 \mathrm{pm} /{ }^{\circ} \mathrm{C}$. Therefore, it can be applied as a temperature sensor.

\section{References}

[1] Kopp V.I., Churikov V.M., Singer J., Chao N., Neugroschl D., Genack A.Z., Chiral fiber gratings, Science 305(5680), 2004, pp. 74-75, DOI: 10.1126/science.1097631.

[2] Shin W., Yu B.A., Noн Y.C., LeE J., Ko D.K., Он K., Bandwidth-tunable band-rejection filter based on helicoidal fiber grating pair of opposite helicities, Optics Letters 32(10), 2007, pp. 1214-1216, DOI: 10.1364/OL.32.001214.

[3] Churikov V.M., Kopp V.I., Genack A.Z., Chiral diffraction gratings in twisted microstructured fibers, Optics Letters 35(3), 2010, pp. 342-344, DOI: 10.1364/OL.35.000342.

[4] Wong K.L.G., Xi X., Kang M.S., Lee H.W., Biancalana F., Conti C., Weiss T., Russell P.St.J., Excitation of orbital angular momentum resonances in helically twisted photonic crystal fiber, Science 337(6093), 2012, pp. 446-449, DOI: 10.1126/science.1223824.

[5] GaO R., JiAng Y., JiAng L., Multi-phase-shifted helical long period fiber grating based temperature-insensitive optical twist sensor, Optics Express 22(13), 2014, pp. 15697-15709, DOI: 10.1364/ OE.22.015697.

[6] Zhang L., LiU Y., ZhaO Y., WANG T., High sensitivity twist sensor based on helical long-period grating written in two-mode fiber, IEEE Photonics Technology Letters 28(15), 2016, pp. 1629-1632, DOI: $10.1109 /$ LPT.2016.2555326.

[7] Zhang H.L., Zhang W.G., Chen L., Xie Z.D., Zhang Z., Yan T.Y., Wang B., Bidirectional torsion sensor based on a pair of helical long-period fiber gratings, IEEE Photonics Technology Letters 28(15), 2016, pp. 1700-1702, DOI: 10.1109/LPT.2016.2533478.

[8] Wang P., Li H., Helical long-period grating formed in a thinned fiber and its application to a refractometric sensor, Applied Optics 55(6), 2016, pp. 1430-1434, DOI: 10.1364/AO.55.001430.

[9] Bing S., Wei W., Liao C.R., Zhang L., Zhang Z.X., Chen M.Y., Wang Y.P., Automatic arc discharge-induced helical long period fiber gratings and its sensing applications, IEEE Photonics Technology Letters 29(11), 2017, pp. 873-876, DOI: 10.1109/LPT.2017.2693361.

[10] XIAn L.L., WANG P., Li H.P., Power-interrogated and simultaneous measurement of temperature and torsion using paired helical long-period fiber gratings with opposite helicities, Optics Express 22(17), 2014, pp. 20260-20267, DOI: 10.1364/OE.22.020260.

[11] Fu C.L., Liu S., Bai Z.Y., He J., Liao C.R., Wang Y., Li Z., Zhang Y., Yang K., Yu B., Wang Y., Orbital angular momentum mode converter based on helical long period fiber grating inscribed 
by hydrogen-oxygen flame, Journal of Lightwave Technology 36(9), 2018, pp. 1683-1688, DOI: 10.1109/JLT.2017.2787120.

[12] Zhao H., Wang P., YamaKawa T., Li H.P., All-fiber second-order orbital angular momentum generator based on a single-helix helical fiber grating, Optics Letters 44(21), 2019, pp. 5370-5373, DOI: $10.1364 /$ OL.44.005370.

[13] Oh S., Lee K.R., Paek U.C., Chung Y., Fabrication of helical long-period fiber gratings by use of a $\mathrm{CO}_{2}$ laser, Optics Letters 29(13), 2004, pp. 1464-1466, DOI: 10.1364/OL.29.001464.

[14] JÁuREGUI C., LóPeZ-Higuera J.M., Virtual long-period gratings, Optics Letters 30(1), 2005, pp. 14-16, DOI: $10.1364 /$ OL.30.000014.

[15] Zhang Y., Bai Z., Fu C., Liu S., Tang J., Yu J., Liao C., Wang Y., He J., Wang Y., Polarization-independent orbital angular momentum generator based on a chiral fiber grating, Optics Letters 44(1), 2019, pp. 61-64, DOI: 10.1364/OL.44.000061.

[16] Fu C.L., Wang Y.P., Bai Z.Y., Liu S., Zhang Y., Li Z., Twist-direction-dependent orbital angular momentum generator based on inflation-assisted helical photonic crystal fiber, Optics Letters 44(2), 2019, pp. 459-462, DOI: 10.1364/OL.44.000459.

[17] Michie A., Canning J., Bassett I., Haywood J., Digweed K., Åslund M., Ashton B., Stevenson M., Digweed J., Lau A., Scandurra D., Spun elliptically birefringent photonic crystal fibre, Optics Express 15(4), 2007, pp. 1811-1816, DOI: 10.1364/OE.15.001811.

[18] Kopp V.I., Churikov V.M., Zhang G., Singer J., Draper C.W., Chao N., Neugroschl D., Genack A.Z., Single- and double-helix chiral fiber sensors, Journal of the Optical Society of America B 24(10), 2007, pp. A48-A52, DOI: 10.1364/JOSAB.24.000A48.

[19] Ivanov O.V., Propagation and coupling of hybrid modes in twisted fibers, Journal of the Optical Society of America A 22(4), 2005, pp. 716-723, DOI: 10.1364/JOSAA.22.000716.

[20] Shvets G., Trendafilov S., Kopp V.I., Neugroschl D., Genack A.Z., Polarization properties of chiral fiber gratings, Journal of Optics A: Pure and Applied Optics 11(7), 2009, article 074007, DOI: 10.1088/1464-4258/11/7/074007.

[21] ERdogan T., Fiber grating spectra, Journal of Lightwave Technology 15(8), 1997, pp. 1277-1294, DOI: $\underline{10.1109 / 50.618322}$. 